This is a postprint of an article published in

Niemann, H.H., Petoukhov, M.V., Härtlein, M., Moulin, M., Gherardi, E., Timmins, P., Heinz, D.W., Svergun, D.I.

$X$-ray and Neutron Small-Angle Scattering Analysis of the Complex Formed by the Met Receptor and the Listeria monocytogenes Invasion Protein InIB

(2008) Journal of Molecular Biology, 377 (2), pp. 489-500. 
Met/InlB complex in solution

\section{X-ray and neutron small-angle scattering analysis of the complex formed by the Met receptor and the Listeria monocytogenes invasion protein InIB}

Hartmut H. Niemann ${ }^{1,2+}$, Maxim V. Petoukhov ${ }^{3,4+}$, Michael Härtlein ${ }^{5}$, Martine Moulin $^{5}$, Ermanno Gherardi ${ }^{6}$, Peter Timmins $^{5}$, Dirk W. Heinz ${ }^{1^{*}}$, and Dmitri Svergun $^{3,4^{*}}$

${ }^{1}$ Division of Structural Biology, Helmholtz Centre for Infection Research, Inhoffenstraße 7, D-38124 Braunschweig, Germany

${ }^{2}$ Department of Chemistry, Bielefeld University, Universitätsstraße 25, 33615 Bielefeld, Germany

${ }^{3}$ European Molecular Biology Laboratory, Hamburg Outstation, Notkestrasse 85, 22603 Hamburg, Germany

${ }^{4}$ Institute of Crystallography, Russian Academy of Sciences, Leninsky pr. 59, 117333 Moscow, Russia;

${ }^{5}$ Institut Laue-Langevin, 6 rue Jules Horowitz, 38042 Grenoble Cedex 9, France

${ }^{6}$ MRC Centre, Hills Road, Cambridge CB2 2QH, United Kingdom

${ }^{+}$These authors contributed equally

* Corresponding authors

Running Title: Met/InlB complex in solution 


\section{Summary}

The Listeria monocytogenes surface protein InIB binds to the extracellular domain of the human receptor tyrosine kinase (RTK) Met, the product of the c-met proto-oncogene. InIB binding activates the Met receptor leading to uptake of Listeria into normally non-phagocytic host cells. The N-terminal half of InlB

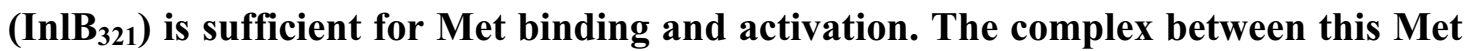
binding domain of InIB and various constructs of the Met ectodomain was characterized by size exclusion chromatography and dynamic light scattering and structural models were built using small angle X-ray and neutron scattering (SAXS and SANS). Although most RTK ligands induce receptor dimerization, InIB 321 consistently binds the Met ectodomain with a 1:1 stoichiometry. A construct comprising the Sema and PSI domains of Met, although sufficient to bind the physiological Met ligand hepatocyte growth factor / scatter factor (HGF/SF), does not form a complex with $\mathrm{InIB}_{321}$ in solution, highlighting the importance of Met Ig domains for InIB-binding. SAXS and SANS measurements of ligand and receptor, both free and in the complex, reveal an elongated shape for the receptor. The four Ig domains form a bent rather than fully extended conformation, and InIB $_{321}$ binds to Sema and the first Ig domain of Met, in agreement with the recent crystal structure of a smaller Met fragment in complex with InlB $_{321}$. These results call into question, whether receptor dimerization is the basic underlying event in InIB $_{321}$ mediated Met activation and demonstrate differences in the mechanisms by which the physiological ligand HGF/SF and $\mathrm{InIB}_{321}$ bind and activate the Met receptor. 
Met/InlB complex in solution

\section{Keywords:}

Complex formation

Rigid body modeling

Neutron scattering

Receptor tyrosine kinase

Small angle scattering

\section{Abbreviations used:}

DLS, dynamic light scattering; HFG/SF, hepatocyte growth factor / scatter factor; IR, inter-repeat; LRR leucine-rich repeat; MM, molecular mass; NSD, normalized spatial discrepancy; SAS, small angle scattering; SANS small angle neutron scattering; SAXS, small angle X-ray scattering; SEC, size exclusion chromatography, 
Met/InlB complex in solution

\section{Introduction}

The gram-positive pathogen Listeria monocytogenes is the causative agent of human listeriosis, a severe disease affecting mainly immunocompromised individuals and manifesting in e.g. diarrhea, meningitis or fetal death. ${ }^{1}$ As a facultative intracellular pathogen, L. monocytogenes is not only able to survive and replicate in the cytoplasm of host cells, but it actively induces its own uptake into normally non-phagocytic cells. ${ }^{2}$ So far, two proteins, InlA - or internalin - and InlB, have been described that on their own are able to stimulate bacterial uptake by binding to specific host cell receptors. InlA is required for crossing the intestinal barrier following ingestion of bacteria with contaminated food. It induces uptake into epithelial cells expressing the InlA receptor Ecadherin. ${ }^{3 ;}$ In contrast, systemic infection requires InlB, a second surface protein of $L$. monocytogenes that induces uptake into a broader range of host cells expressing the InlB receptor Met, including endothelial cells and hepatocytes. $5 ; 6$

Met is a receptor tyrosine kinase with hepatocyte growth factor / scatter factor (HGF/SF) as its natural ligand. ${ }^{7} \mathrm{HGF} / \mathrm{SF}$ induced Met signaling promotes a complex cellular response including the stimulation of cell division and cell migration. Both proteins are essential during embryonic development. In the adult, Met signaling is involved in wound healing and its deregulation is implicated in tumor metastasis. Binding of ligands to the Met extracellular domain (ectodomain) causes phosphorylation of its cytoplasmic tyrosine kinase domain, which in turn facilitates the interaction with numerous downstream effectors of signaling. ${ }^{8}$ 
Met/InlB complex in solution

InlB is a 630 amino acid multi-domain surface protein that belongs to the internalin family of L. monocytogenes. ${ }^{9}$ The function of individual domains for binding and activation of Met has been characterized in depth. A fragment consisting only of the N-terminal Cap region and the leucine-rich repeat (LRR) region is sufficient for high affinity binding of $\mathrm{Met}^{6}$ and can induce Met activation when it is artificially dimerized by a disulfide-bridge ${ }^{10}$ or clustered by immobilization on latex beads. ${ }^{11}$ InlB $_{321}$, additionally containing the so-called inter-repeat (IR) region is the smallest InlB fragment that promotes Met phosphorylation as a soluble, monomeric protein. ${ }^{10}$ The IR region is followed by a poorly characterized B-repeat and three C-terminal, highly basic GW domains that do not bind Met on their own, ${ }^{10}$ but significantly enhance Met phosphorylation and are required for a full cellular response. ${ }^{10 ; 12}$ The synergistic effect of the $\mathrm{N}$ - and C-terminal InlB domains depends on the presence of heparan sulfate proteoglycans (HSPGs) on the host cell surface ${ }^{10 ; 12}$ and the GW domains strongly interact with negatively charged heparin in vitro, resulting in clustering of InlB. ${ }^{13}$ Several crystal structures of InlB fragments ${ }^{14 ; 15}$ and the full-length protein ${ }^{16}$ have revealed that the Cap, the LRR and the IR region form a rigid unit with a single hydrophobic core, the so called internalin domain. The Met binding site has been mapped to the concave face of the kidney shaped internalin domain of InlB, as point mutation to Ser of aromatic side chains exposed on the concave surface impair receptor binding. ${ }^{17}$

The Met extracellular region consists of six domains ${ }^{7}$. The N-terminal Sema domain, a seven bladed $\beta$-propeller containing the binding site for $\mathrm{HGF} / \mathrm{SF}^{18 ;}$, is followed by a small, cysteine-rich PSI domain and four immunoglobulin-like Ig domains. Here we have studied the binding of InlB $_{321}$ in solution to the whole Met ectodomain 
Met/InlB complex in solution

(Met $\left.{ }_{928}\right)$ and two shorter Met fragments (Met ${ }_{838}$ lacking the C-terminal and Met $_{567}$ lacking all Ig-like domains). These studies complement a concomitant crystal structure of InlB $_{321}$ bound to Met ${ }_{741}$, a Met fragment lacking two of the Ig-like domains ${ }^{13}$ and offer independent insights on the interactions between InlB and Met.

The stoichiometry and quaternary structure of Met-InlB complexes in solution was studied by size exclusion chromatography (SEC), dynamic light scattering (DLS) and small-angle scattering (SAS). The latter method is widely used to study the structure of biological macromolecules at a resolution of 1-2 $\mathrm{nm}$, and it is applicable to a broad range of sizes, from individual macromolecules to multi-domain proteins and large macromolecular assemblies. ${ }^{20}$ Recent progress in instrumentation and especially development of novel data analysis methods ${ }^{21}$ significantly enhanced resolution and reliability of structural models provided by the technique and made it possible to effectively use a combination of small-angle X-ray and neutron scattering (SAXS and SANS). Indeed, the absence of radiation damage and possibility of contrast variation with hydrogen/deuterium exchange makes SANS an extremely useful complementary tool to SAXS with its high brilliance and rapid data collection. The additional information gained by contrast variation using $\mathrm{H}_{2} \mathrm{O} / \mathrm{D}_{2} \mathrm{O}$ mixtures but also deuteration of individual components (for example, see ${ }^{22}$ ) is especially important in the study of functional complexes. A SAXS/SANS combination was successfully employed in the past to study objects like the ribosome. ${ }^{23}$ Recently, novel approaches have been developed to use SAXS/SANS for structural characterization of multi-component complexes, ${ }^{24 ;} 25$ and these methods are applied in this study to elucidate the structure of Met-InlB complexes in solution. 
Met/InlB complex in solution

\section{Results}

\section{InIB ${ }_{321}$ and the Met ectodomain form a stable 1:1 complex in solution}

The purified full-length Met ectodomain $\left(\right.$ Met $\left._{928}\right)$ produced in glycosylation deficient $\mathrm{CHO}$ lec8 cells forms a stable complex with $\mathrm{InlB}_{321}$ that can be purified by SEC. Met ${ }_{928}$ binds one molar equivalent completely, while a second molar equivalent remains quantitatively unbound, indicating a strict 1:1 stoichiometry (Fig. 1). This conclusion is confirmed by careful titration showing that any $\operatorname{InlB}_{321}$ added beyond 1 molar equivalent elutes from the column as free protein (data not shown).

The elution volume of Met $_{928}$ corresponds to a molecular weight of approximately $210 \mathrm{kDa}$ when compared with globular standard proteins against a calculated mass of 102 $\mathrm{kDa}$ for the polypeptide chain alone and $117 \mathrm{kDa}$ including N-linked glycans (H.H. Niemann and M. Nimtz, unpublished results). Met ${ }_{928}$, however, behaves as a monomer in solution with an estimated MM of $109 \mathrm{kDa}$ by analytical ultracentrifugation ${ }^{18}$ and has a non-globular, elongated shape ${ }^{26}$. Therefore, the observed peak most likely corresponds to a Met monomer. Binding of $\operatorname{InlB}_{321}(32 \mathrm{kDa})$ increases the apparent MM by some $25 \mathrm{kDa}$ indicating that one molecule of $\mathrm{InlB}_{321}$ binds to monomeric $\mathrm{Met}_{928}$, and that ligand binding does not induce dimerization of the receptor ectodomain in vitro. This conclusion is backed by data from DLS (Table 1) and SAS (see below). 
Met/InlB complex in solution

\section{The N-terminal half of the Met stalk contains the high affinity binding site for InIB}

SEC and DLS were repeated with C-terminally truncated constructs of the Met ectodomain lacking either domain Ig4 (Met 838$)$ or domains Ig3 and Ig4 (Met $\left.{ }_{741}\right)$. The results show that $\operatorname{Ig} 3$ and $\operatorname{Ig} 4$ are dispensable for binding and that both truncated constructs still form a stable 1:1 complex with $\operatorname{InlB}_{321}$ (Table 1). This is consistent with the results of solid-phase binding assays showing that Met $_{741}$ and Met ${ }_{838}$ have the same affinity for $\operatorname{InlB}_{321}$ as Met $_{928}{ }^{13}$. An even shorter construct (Met ${ }_{567}$ ) consisting only of the Sema and PSI domains and lacking all four Ig domains did not form a complex with $\mathrm{InlB}_{321}$. In SEC runs, both proteins eluted as single peaks with elution volumes identical to the free protein, showing no sign of interaction (Fig 2 and Table 1). In dynamic light scattering, the mixture of $\operatorname{Met}_{567}\left(62 \mathrm{kDa}\right.$ without sugars) and $\operatorname{InlB}_{321}$ had a hydrodynamic radius intermediate to that of the free proteins, indicating a mere mixture rather than a complex (Table 1). Again, this confirms data from solid-phase binding assays ${ }^{13}$ and demonstrates that Ig1 and/or Ig2 of the Met stalk are essential for high affinity binding of $\mathrm{InlB}_{321}$. The relative contribution of these two domains, however, could not be conclusively established by deletion mutagenesis because so far, we have not been able to purify Met $_{656}$, a construct consisting of the Sema, PSI and only the first Ig-like domain.

\section{Overall parameters from SAXS}

The experimental X-ray scattering patterns from Met constructs, free $\mathrm{InlB}_{321}$ and their complexes are presented in Fig. 3(a), as the logarithms of the scattering intensity I versus momentum transfer $s=4 \pi \sin (\theta) / \lambda$, where $2 \theta$ is the scattering angle and $\lambda$ is the 
Met/InlB complex in solution

wavelength of the radiation. The overall structural parameters computed from the SAXS data are given in Table 2. The molecular masses $(M M)$ and the volumes $\left(V_{p}\right)$ of all individual macromolecules (various Met constructs and free $\mathrm{InlB}_{321}$ ) suggest monomeric states of all these solutes. The values obtained for the equimolar mixture of $\mathrm{Met}_{567}$ and InlB $_{321}$ are smaller than those predicted for monomeric Met ${ }_{567}$, which demonstrates that no stable complex formation takes place and the two species remain unbound. The $M M s$ and the $V_{p}$ values of the two largest Met constructs (Met ${ }_{838}$ and Met ${ }_{928}$ ) mixed with $\mathrm{InlB}_{321}$ reveal a significant increase compared to the unbound state, compatible with the formation of a 1:1 complex. This finding suggests that some of the first three Ig-like domains play an important role in binding of $\mathrm{InlB}_{321}$. On the other hand, the maximum diameter $\left(D_{\max }\right)$ values of the complexes are practically unchanged and their radii of gyration are even smaller than those of free Met $_{838}$ and Met ${ }_{928}$. This suggests that the constructs keep their extended conformation in the complex and that the $\mathrm{InlB}_{321}$ is bound to their central parts.

The scattering curves from the atomic models of $\mathrm{InlB}_{321}$ and of $\mathrm{Met}_{567}$ with added sugars computed by CRYSOL ${ }^{27}$ agree reasonably well with the corresponding SAXS profiles (Fig. 3(a)) with discrepancy $\chi=1.6$ and 1.4, respectively (see definition of discrepancy in Methods). This validates the monomeric state of the proteins in solution and structural similarity with their crystallographic models. The experimental data of the Met $_{567}+$ InlB $_{321}$ mixture can be fitted by a linear combination of the two individual solutes with the program OLIGOMER ${ }^{28}$ with $\chi=1.1$ (Fig. 3(a)) further confirming that no complex is formed between $\mathrm{Met}_{567}$ and $\mathrm{InlB}_{321}$. 
Met/InlB complex in solution

\section{Molecular modeling}

No variation of the maximum sizes of $\mathrm{Met}_{838}$ and $\mathrm{Met}_{928}$ upon $\mathrm{In}_{1 \mathrm{~B}} \mathrm{~B}_{21}$ binding was observed and moreover the difference in $D_{\max }$ between Met ${ }_{838}$ and Met $_{928}$ (and between the corresponding complexes) is compatible with the addition of one Ig-like domain at the periphery of the Met molecule. The SANS scattering profiles from free Met $_{928}$ and from its complex with $50 \%$ deuterated $\mathrm{InlB}_{321}$ at $81 \% \mathrm{D}_{2} \mathrm{O}$ (the isoscattering point of $50 \%$ deuterated protein at which the scattering from $\mathrm{InlB}_{321}$ is matched out) were very similar suggesting no gross conformational changes of the Met ectodomain upon ligand binding. Rigid body molecular modeling was performed based on the crystallographic models of $\operatorname{InlB}_{321}{ }^{15}$ and $\operatorname{Met}_{567}{ }^{19}$ and on homology models of the four Ig-like domains ${ }^{18}$ assuming a similar overall arrangement of the domains in both Met constructs and their complexes. The program SASREF ${ }^{24 ; 25}$ was employed to construct a low resolution 3D model of the Met $_{928}+\mathrm{InlB}_{321}$ complex from six high resolution models of the subunits (Met $\left.567, \operatorname{Ig} 1, \operatorname{Ig} 2, \operatorname{Ig} 3, \operatorname{Ig} 4, \operatorname{InlB}_{321}\right)$. The algorithm simultaneously fitted a total of 32 solution scattering curves from the truncated and the full length Met and the corresponding complexes (4 SAXS curves and 28 SANS contrast variation data with protonated, $50 \%$ and $100 \%$ deuterated InlB $_{321}$ in Fig. 3(b), Table 3). Multiple SASREF runs were performed and in all reconstructions the information about the Met-binding interface of InlB (the four aromatic residues: F104, W124, Y170 and Y214 ${ }^{17}$ ) was included in the modeling as a soft restraint. The requirement of Met interconnectivity in terms of proximity of appropriate $\mathrm{C}$ - and $\mathrm{N}$ - termini of the domains was also used. The multiple reconstruction attempts converged to two families of models yielding similar overall agreement with the experimental data. In models of the first family both the Sema 
Met/InlB complex in solution

and the Ig-1 domains of Met interact with InlB, in models of the second family only the Sema domain contacts the ligand. The models within both families were compared with each other by the program DAMAVER ${ }^{29}$ which also provided the most typical representatives. The averaged normalized spatial discrepancy (NSD) of 1.35 was obtained for the first group, 1.31 for the second whereas the discrepancy between their representatives was 1.81 (NSD is a measure of dissimilarity between two threedimensional models, and lower NSD points to better agreement). The second family of models could be discarded based on the SEC, DLS and SAXS data of Met ${ }_{567}+\operatorname{InlB}_{321}$ mixtures that clearly implied a requirement for at least one Ig domain for InlB binding (Fig. 2 and Tables 1 and 2). The final SAS-based low resolution 3D model of the complex selected by DAMAVER (Fig. 4) yields good fits ( $\chi$ values given in table 3 ) to all scattering curves presented in Fig. 3 with the overall $\chi=2.3$. The Met M28 portion of this model is also in a good agreement $(\mathrm{NSD}=1.43)$ with the solution structure of the Met ectodomain reported earlier in the study of its interactions with $\mathrm{HGF} / \mathrm{SF}^{26}$

This model was built from high resolution models of individual subunits using only solution scattering data and biochemical information about the InlB Met-binding site and independently from crystallization efforts that were carried out in parallel. The complex between the shorter Met $_{741}$ construct lacking the last two Ig domains and $\operatorname{InlB}_{321}$ has recently been crystallized and its structure has been solved. ${ }^{13}$ It displays the Met-InlB complex with both the Met Sema and Ig1 domains forming contacts to $\operatorname{InlB}_{321}$ in agreement with the SAS model. The two models are superimposed in Fig. 4 and the overall appearance of the complex is similar. The $\mathrm{Met}_{741} / \mathrm{InlB}_{321}$ fragment taken from the SAS model yields an NSD=1.38 to the crystal structure (comparable with the 
Met/InlB complex in solution

averaged NSD of 1.35 between the SAS models of the first type). The positions of most domains in the crystal structure are matched reasonably well by those of the SAS rigid body model as seen in Fig 4, with the exception of the small PSI domain. More pronounced differences are found with respect to orientation for $\operatorname{InlB}_{321}$ (Cap and IR are flipped) and for the propeller of the Sema domain reflecting the limitations of the SAS method caused by its low resolution.

To further reconcile the crystal structure of the $\mathrm{Met}_{741} / \mathrm{InlB}_{321}$ complex with the solution scattering data, a rigid body modeling approach against the same data set of 32 curves was applied to define the last two Ig-like domains missing in the crystallographic model. In this approach, $\mathrm{Met}_{567}, \mathrm{InlB}_{321}$ and the first Ig domain were fixed, whereas the second Ig-like domain was refined in the vicinity of its position in the crystal structure and the third and the fourth Ig-like domains were placed so that the entire Met molecule is interconnected and the overall fit to the solution scattering data is optimal. As seen in Fig. 5 the refined crystallographic model with the two C-terminal Ig domains added is similar to the original SAS-based one, except for the positioning of the PSI domain and the above mentioned rotational ambiguity. The crystal structure-based model has a slightly higher overall discrepancy to the SAS data with $\chi=2.9$ (data not shown). The higher deviations could be explained either by small conformational changes of Met upon complex formation or by minor structure dissimilarities between crystal and solution due to crystal packing forces. 
Met/InlB complex in solution

\section{Discussion}

In this study, we have characterized the complex formed in solution between the human Met ectodomain and the Met binding domain of the bacterial invasion protein InlB using purified, recombinant protein. We could derive several characteristics of the complex directly from SAS measurements without a priori information, while the subsequent rigid body modeling required biochemical and structural information. The first clear result obtained from the SAXS/SANS data alone is that Met ${ }_{928}$ and $\operatorname{InlB}_{321}$ form a 1:1 rather than a 2:2 complex. This finding is backed by less direct evidence from size exclusion chromatography and dynamic light scattering and has considerable biological implications, which will be discussed below.

Secondly, we find that the longest molecular axis of Met does not increase upon ligand binding, and even more, the radius of gyration decreases. Hence, InlB does not bind the region of Met projecting furthest away from the host-cell surface, but rather associates laterally with an elongated receptor molecule. Given the domain organization of the Met extracellular region (an N-terminal disc-shaped Sema domain and an elongated C-terminal stalk of four Ig-like domains), this result strongly suggests a lateral association involving the stalk. This behavior contrasts sharply with that of the natural ligand $\mathrm{HGF} / \mathrm{SF}$. Binding of $\mathrm{HGF} / \mathrm{SF}$ to $\mathrm{Met}_{928}$ increases both the radius of gyration and the maximum size. ${ }^{26}$ Accordingly, no interaction between Met Ig domains and HGF/SF has been described. Instead, HGF/SF binds the Met Sema domain, namely the propeller face pointing away from the Met stalk as apparent from a crystal structure of the HGF/SF $\beta$-chain in complex with the Met Sema and PSI domain and additional electron 
Met/InlB complex in solution

microscopy and SAXS studies of complete $\mathrm{HGF} / \mathrm{SF}$ in complex with the whole Met ectodomain. ${ }^{19 ; 26}$

Third, SAXS measurements with Met $_{567}$ (lacking all four Ig-like domains) have unequivocally shown that this construct does not form a complex with $\operatorname{InlB}_{321}$. Again these data are supported by the results from SEC and DLS presented here. Hence, one or several of the Met Ig-like domains are critical for binding of InlB, supporting the idea of InlB associating laterally with the Met stalk. Furthermore, the study clearly demonstrates that neither the $3^{\text {rd }}$ or $4^{\text {th }} \mathrm{C}$-terminal Ig-like domain of Met are required for InlB binding, as shown by SAS experiments with Met $_{838}$, a construct lacking Ig4 and by SEC and DLS with Met $_{741}$.

Ligand induced dimerization is generally thought to be the underlying event in activation of receptor tyrosine kinases ${ }^{30}$ and dimerization of the Met ectodomain is apparently sufficient to drive Met activation. ${ }^{10}$ However, small angle scattering provides no evidence for dimerization of the Met ectodomain (or fragments of it) either alone or in complex with $\operatorname{InlB}_{321}$. This is in agreement with data from analytical ultracentrifugation ${ }^{13}$. Notably, the SAS data presented here have been measured at concentrations of up to $40 \mathrm{mg} / \mathrm{ml}$, while analytical ultracentrifugation had been carried out at approx. $0.3 \mathrm{mg} / \mathrm{ml}$. These data contrast with other reporting a high affinity selfinteraction of the soluble Met ectodomain with immobilized Met in vitro. ${ }^{31}$ In a physiological context, auxiliary factors not present in our in vitro system may promote $\mathrm{InlB}_{321}$ induced Met dimerization on the cell surface. This issue, however, needs to be clarified by other methods. 
Met/InlB complex in solution

A low-resolution model of the complex was constructed using available highresolution structures and homology models of individual components under the assumption that the structure of Met does not change upon complex formation. This assumption seemed justified given that all the available SAXS and SANS data from free Met, $\mathrm{InlB}_{321}$ and their complexes can be reconciled by a single model. Moreover, the similar SANS curves of free Met $_{928}$ and Met $_{928}$ bound to $50 \%$ deuterated $\operatorname{InlB}_{321}$ at the isoscattering point of $50 \%$ deuterated protein suggest that there are no major conformational changes of Met upon binding of $\mathrm{InlB}_{321}$. Nevertheless, electron microscopy has shown that in the absence of ligand, the Met ectodomain is flexible. ${ }^{26}$ This is corroborated by the fact that the relative orientation of the Sema and PSI domains is dramatically different in the crystal structures of $\mathrm{Met}_{567}$ in complex with HGF/SF and Met $_{741}$ in complex with InlB $_{321} .{ }^{13 ; 19}$ The lack of proof for a major conformational change in the SAS measurements suggests that the actual rearrangement upon binding of $\operatorname{InlB}_{321}$ to free Met in solution may not be as dramatic as the 60 degree rotation of the Sema domain that marks the difference between the structure of HGF/SF-bound Met and InlB $_{321}$-bound Met. However, smaller conformational changes, which may well be sufficient for receptor activation, will not be resolved by a low-resolution method like SAS. Moreover, SAS averages over all conformations in solution, and hence will not resolve structural heterogeneity such as that suggested by electron microscopy studies of the Met ectodomain. ${ }^{26}$ Thus, the defined InlB $_{321}$-bound conformation of the ectodomain of Met may be similar to the average of an ensemble of conformations of free Met.

The Met binding site for InlB in the final model based on the SAS data predicts an interaction between InlB and the Met Sema domain, although this interaction was not 
Met/InlB complex in solution

suggested by binding data of Met $_{567}$ and has, therefore, not been input as a modeling restraint. An interaction between InlB and the Met Sema domain is also present in two independent crystal structures of Met $_{741}$ complexed with $\operatorname{InlB}_{321}{ }^{13}$ The overall appearance of the SAS-derived complex is largely compatible with the structure of the shorter crystallographic construct although e.g. the orientation of $\operatorname{InlB}_{321}$ in the SASderived model is different from that in the crystal structures. Hence, InlB ${ }_{321}$ contacts Sema with its Cap and not with its IR region as found in the crystal structures. This may reflect an intrinsic limitation of SAS as a low-resolution method. Indeed, InlB is an elongated and rather symmetric molecule and its rotations yield little variations to the scattering from the complex (even to multiple scattering patterns at different contrasts). In principle, orientational ambiguity can be resolved e.g. by using SAS together with residual dipolar coupling data from NMR to establish relative orientations of domains. ${ }^{32}$; $33 ; 34$

The SAXS/SANS study of Met-InlB interactions in solution yields a comprehensive picture of the complex formation in solution. Although the SAS model is not quite identical to the crystal structure, the SAS model also revealed prominent features like the interaction of $\operatorname{InlB}_{321}$ with both the Sema and the Ig1 domain of Met. The SAS data further allowed modeling the last two Ig-like domains of the Met stalk in the $\mathrm{InlB}_{321}$-Met complex and, even more importantly, the SAS results provide insights into the Met-InlB interactions and oligomerization behavior in solution. These results further underline the great potential of the SAXS-SANS methodology and demonstrate that this approach can provide unique results about the structural organization of functional macromolecular complexes. 
Met/InlB complex in solution 
Met/InlB complex in solution

\section{Materials and Methods}

\section{Expression of deuterium-labeled InIB ${ }_{321}$ for neutron scattering}

For protein expression in fully (perdeuterated) and 50\% deuterated media, codons 36-321 of the Listeria monocytogenes InlB gene were cloned into vector pETM30 providing an N-hexa-His-GST tag followed by a TEV cleavage site (G.Stier, EMBL, Heidelberg, Germany) that confers kanamycin resistance. Kanamycin resistance allows efficient selection for the expression construct under High Cell Density conditions in deuterated minimal media. Deuterium labeled $\mathrm{InlB}_{321}$ was obtained by heterologous expression in Escherichia coli BL21(DE3) CodonPlus (Stratagen) at the ILL-EMBL Deuteration Facility in Grenoble, France. Cells were grown in minimal medium: $6.86 \mathrm{~g} \mathrm{~L}$ ${ }^{-1}\left(\mathrm{NH}_{4}\right)_{2} \mathrm{SO}_{4}, 1.56 \mathrm{~g} \mathrm{~L}^{-1} \mathrm{KH}_{2} \mathrm{PO}_{4}, 6.48 \mathrm{~g} \mathrm{~L}^{-1} \mathrm{Na}_{2} \mathrm{HPO}_{4} 2 \mathrm{H}_{2} \mathrm{O}, 0.49 \mathrm{~g} \mathrm{~L}^{-1}$ diammonium hydrogen citrate, $0.25 \mathrm{~g} \mathrm{~L}^{-1} \mathrm{MgSO}_{4} \cdot 7 \mathrm{H}_{2} \mathrm{O}, 1.0 \mathrm{ml} \mathrm{L}^{-1}\left(0.5 \mathrm{~g} \mathrm{~L}^{-1} \mathrm{CaCl}_{2} \cdot 2 \mathrm{H}_{2} \mathrm{O}, 16.7 \mathrm{~g} \mathrm{~L}^{-1}\right.$ $\mathrm{FeCl}_{3} \cdot 6 \mathrm{H}_{2} \mathrm{O}, 0.18 \mathrm{~g} \mathrm{~L}^{-1} \mathrm{ZnSO}_{4} \cdot 7 \mathrm{H}_{2} \mathrm{O}, 0.16 \mathrm{~g} \mathrm{~L}^{-1} \mathrm{CuSO}_{4} \cdot 5 \mathrm{H}_{2} \mathrm{O}, 0.15 \mathrm{~g} \mathrm{~L}^{-1} \mathrm{MnSO}_{4} \cdot 4 \mathrm{H}_{2} \mathrm{O}$, $0.18 \mathrm{~g} \mathrm{~L}^{-1} \mathrm{CoCl}_{2} 6 \mathrm{H}_{2} \mathrm{O}, 20.1 \mathrm{~g} \mathrm{~L}^{1}$ EDTA), $5 \mathrm{~g} \mathrm{~L}^{-1}$ glycerol, $40 \mathrm{mg} \mathrm{L}^{-1}$ kanamycin, $34 \mathrm{mg}$ $\mathrm{L}^{-1}$ chloramphenicol. ${ }^{35 ; 36}$ For preparation of fully deuterated medium, mineral salts were dried out in a rotary evaporator (Heidolph) at $333 \mathrm{~K}$ temperature and labile protons were exchanged for deuterons by dissolving in a minimal volume of $\mathrm{D}_{2} \mathrm{O}$ and then drying again. Perdeuterated $d_{8}$-glycerol (Euriso-Top, France) was used as a carbon source. For preparation of $50 \%$ deuterated medium $\mathrm{H}_{2} \mathrm{O}$ and $\mathrm{D}_{2} \mathrm{O}$ as well as $\mathrm{d}_{8}$-glycerol and unlabeled glycerol were mixed in a ratio 1:1 volume to volume.

Adaptation of BL21(DE3) cells to deuterated minimal medium was achieved by a multi-stage adaptation process. ${ }^{36}$ For High Cell Density cultivation, $1.5 \mathrm{~L}$ of deuterated 
Met/InlB complex in solution

medium was inoculated with $100 \mathrm{~mL}$ pre-culture of adapted cells in a $3 \mathrm{~L}$ fermenter (Labfors, Infors). During the batch and fed-batch phases the pH was adjusted to 6.9 (by addition of $\mathrm{NaOD}$ ) and the temperature was adjusted to $303 \mathrm{~K}$. The gas-flow rate of sterile filtered air was $0.5 \mathrm{~L} \mathrm{~min}^{-1}$. Stirring was adjusted to ensure a dissolved oxygen tension (DOT) of 30\%. The fed-batch phase was initiated when the optical density at 600 $\mathrm{nm}$ reached 6.0. Glycerol was added to the culture to keep the growth rate stable during fermentation. When $\mathrm{OD}_{600}$ reached 7.2 for the perdeuterated culture or 13.2 for the $50 \%$ deuterated culture, the temperature was decreased to $293 \mathrm{~K}$. GST-InlB 321 over-expression was induced by the addition of IPTG for $2 \mathrm{~h}$ to reach a final concentration of $1 \mathrm{mM}$ and incubation continued for $18 \mathrm{~h}$. Cells were then harvested, washed with $10 \mathrm{mM}$ HEPES ( $\mathrm{pH}$ 6.4), and stored at $193 \mathrm{~K}$. Purification of $\mathrm{InlB}_{321}$ followed a published procedure ${ }^{15}$ except that TEV protease was used to cleave the GST tag.

\section{Protein expression and purification}

Constructs of the human Met ectodomain were expressed in $\mathrm{CHO}$ cells and purified as described. ${ }^{13 ;} 18$ Mass spectrometry revealed the point mutations Y41C and G344A, which were then confirmed by plasmid sequencing. These mutations do not impair binding of the natural ligand HGF/SF or of InlB. InlB for SAXS was expressed and purified as described. ${ }^{15}$ All proteins were purified by SEC on Superdex 200 prior to SAS measurements. Complexes were formed by mixing (for SANS) or by adding a molar excess of InlB $_{321}$ to Met and subsequent purification by SEC (for SAXS). 
Met/InlB complex in solution

\section{Analytical size exclusion chromatography and dynamic light scattering}

All runs were carried out on a Superdex200 HR10/30 column (GE Healthcare) equilibrated in PBS and calibrated with a set of seven standard proteins (GE Healthcare). The samples were applied in a volume of $100 \mu$ containing 0.3 nmole of Met $(0.33$ $\mathrm{mg} / \mathrm{ml}$ for $\mathrm{Met}_{928}$ ) and/or an equimolar amount or a two-fold molar excess of $\mathrm{InlB}_{321}$. DLS measurements were carried out at room temperature in a DynaPro 801 instrument (Protein Solutions) using a $12 \mu \mathrm{l}$ cuvette and protein concentrations between 0.5 and 1 $\mathrm{mg} / \mathrm{ml}$. Hydrodynamic radii are from the regularization data log of the Dynamics software with aqueous buffer as solvent and molecular weights were estimated using the globular protein standard curve.

\section{Small Angle X-ray Scattering}

Synchrotron radiation X-ray scattering data were collected on the X33 camera ${ }^{37 ; 38}$ at the European Molecular Biology Laboratory (EMBL) on the storage ring DORIS III of the Deutsches Elektronen Synchrotron (DESY), using a linear gas detector ${ }^{39}$. Scattering patterns of Met $_{928}$ and the shorter constructs, Met ${ }_{838}$ and Met $_{567}$ lacking the last Ig-like domain or all four of them, respectively, as well as their equimolar mixtures with $\operatorname{InlB}_{321}$ were measured at several (at least three) solute concentrations ranging from 2 to 40 $\mathrm{mg} / \mathrm{ml}$ in $25 \mathrm{mM}$ Na-phosphate buffer, $\mathrm{pH} 7.5,150 \mathrm{mM} \mathrm{NaCl}$. Just prior to measurements, $1 \mathrm{mM}$ DTT was added to reduce the effects of radiation damage. For the sample-to-detector distance of $2.4 \mathrm{~m}$ and the X-ray wavelength $\lambda=0.15 \mathrm{~nm}$ the range of momentum transfer $0.15<s<3.5 \mathrm{~nm}^{-1}$ was covered. 
Met/InlB complex in solution

To check for radiation damage during the scattering experiments, the data were collected in 15 successive 1-minute frames. The individual frames were averaged after normalization to the intensity of the incident beam, corrected for the detector response and the scattering of the buffer was subtracted. The difference curves were scaled for the solute concentration and extrapolated to infinite dilution. All data manipulations were performed using standard procedures by the program package PRIMUS. ${ }^{28}$

The maximum particle dimensions $D_{\max }$ were estimated using the orthogonal expansion program ORTOGNOM ${ }^{40}$. The forward scattering values $I(0)$ and the radii of gyration $\mathrm{R}_{\mathrm{g}}$ were evaluated using the Guinier approximation ${ }^{41}$ assuming that at very small angles $\left(s<1.3 / \mathrm{R}_{\mathrm{g}}\right)$ the intensity is represented as $I(s)=I(0) \exp \left(-\left(s R_{g}\right)^{2} / 3\right)$. These parameters were also computed from the entire scattering patterns using the indirect transform package GNOM. ${ }^{42}$ The molecular masses (MMs) of the solutes were estimated from SAXS data by comparison of the forward scattering with that from reference solutions of bovine serum albumin $(\mathrm{MM}=66 \mathrm{kDa})$.

Due to a limited accuracy of MMs determination by solution scattering influenced by the uncertainty in the measured protein concentrations, the excluded (Porod) volumes of the solutes were also analyzed, taking advantage of the fact that computation of the Porod volume does not depend on data normalization ${ }^{43}$ :

$$
V_{p}=2 \pi^{2} I(0) / \int_{0}^{\infty} s^{2} I(s) d s
$$

For globular proteins, Porod (i.e. hydrated) volumes in $\mathrm{nm}^{3}$ are about twice the MMs in kDa. 
Met/InlB complex in solution

\section{Small angle neutron scattering}

The SANS data were collected on the D22 instrument (http://www.ill.fr/YellowBook/D22/) at the Institut Laue-Langevin, Grenoble, France. Measurements were made in two different instrument configurations: sample-detector distance $5.6 \mathrm{~m}$, neutron wavelength $(\lambda) 8 \AA, \delta \lambda / \lambda=0.1$, for the s-range $0.0067-0.01 \AA^{-1}$ and sample-detector distance $2.0 \mathrm{~m}$, neutron wavelength $(\lambda) 6 \AA, \delta \lambda / \lambda=0.1$, for the srange $0.026-0.42 \AA^{-1}$. Samples were contained in standard quartz cuvettes (Hellma, France) of 1.00 or $2.00 \mathrm{~mm}$ pathlength. Measurements were made on complexes of the bacterial ligand $\operatorname{In}_{1} \mathrm{~B}_{321}$ and the full-length Met ectodomain (Met ${ }_{928}$ ) and its shorter constructs, Met ${ }_{838}$ and Met $_{567}$. Met was always fully protonated and $\operatorname{In}_{1} \mathrm{~B}_{321}$ was either protonated, $50 \%$ deuterated or perdeuterated. For most samples scattering curves were recorded as a contrast variation series in (nominally) $0,35,50,60,80$ and $100 \% \mathrm{D}_{2} \mathrm{O}$. Precise deuterium content of the buffer was estimated by neutron transmission measurements. Sample concentrations were nominally 10 or $5 \mathrm{mg} / \mathrm{ml}$. Data reduction was carried out using standard routines ${ }^{44}$. Briefly, all scattering curves were averaged about the incident beam, corrected for detector response and normalized by the scattering of a $1 \mathrm{~mm}$ water sample. Scattering from buffer and empty cell were then subtracted. The final scattering curves were scaled together by normalizing to unit concentration, cell thickness and sample transmission. The $\mathrm{R}_{\mathrm{g}}$ and $\mathrm{I}(0)$ values were obtained by the Guinier approximation $^{41}$ using standard programs. ${ }^{44}$ 
Met/InlB complex in solution

\section{Molecular Modeling}

The atomic models of the individual subunits were employed for rigid body modeling of the quaternary structure of the solutes. To account for Met glycosylation, the carbohydrate chains were tentatively added to the appropriate asparagines of the available high-resolution structures of Sema and cysteine rich domains (PDB ID 1shy ${ }^{19}$ ) and homology models of four Ig-like domains (1ux3 and 2 cew; $\left.{ }^{18}\right)$. The potential Nglycosylation sites and the structure of attached carbohydrates from $\mathrm{CHO}$ lec 8 cells were analyzed by mass spectrometry. All ten potential N-glycosylation sites were found to be occupied. The attached carbohydrates consist of the basic fucosylated common core structure with zero to four additional other $\mathrm{N}$-acetylglucosamine residues attached in varying ratios (Niemann and Nimtz unpublished data). For the modeling, triantennary structures were added to all $\mathrm{N}$-glycosylation sites $(6,2,1$ and 1 sugar chains were added to Sema, $\operatorname{Ig} 1, \operatorname{Ig} 3$ and Ig4, respectively). A model for triantennary structures was derived from a fucosylated diantenneary structure taken from PDB entry $1 \mathrm{cvi}^{45}$ by addition of a third $\mathrm{N}$-acteylglucosamine. The partial scattering amplitudes of the individual subunits were calculated from their atomic coordinates using the programs CRYSOL for X-rays ${ }^{46}$ and CRYSON ${ }^{47}$ for neutrons. The programs either predict theoretical scattering patterns using default or user-defined parameters or fit the experimental scattering curve by adjusting the excluded volume and the contrast of the hydration layer while minimizing discrepancy:

$$
\chi^{2}=\frac{1}{N-1} \sum_{j}\left[\frac{I_{\mathrm{exp}}\left(s_{j}\right)-c I\left(s_{j}\right)}{\sigma\left(s_{j}\right)}\right]^{2},
$$


Met/InlB complex in solution

where $N$ is the number of experimental points, $c$ is a scaling factor and $\sigma\left(s_{j}\right)$ is the experimental error at the momentum transfer $s_{j}$.

The rigid body modeling of Met-InlB complexes against solution scattering data was performed by the program $\mathrm{SASREF}^{24 ; 25}$. The program employs a simulated annealing protocol to find the positions and orientations of the domains or subunits forming an interconnected assembly without steric clashes while minimizing discrepancy (2) between the calculated and the experimental scattering profiles. If several scattering curves from partial constructs and/or from contrast variation series are available, the program fits these multiple scattering curves simultaneously assuming the same arrangement of the subunits in all constructs/conditions. The scattering intensity from a multi-component particle depends on the contrasts of the components. The contrast is defined as $\Delta \rho=\left\langle\rho(\boldsymbol{r})>-\rho_{s}\right.$, where $\langle\rho(\boldsymbol{r})>$ is the average scattering length density of the component, $\rho_{s}$ is that of the solvent. With neutrons, the former quantity can be changed by selective deuteration, while the latter is conveniently varied in a broad range by using $\mathrm{H}_{2} \mathrm{O} / \mathrm{D}_{2} \mathrm{O}$ mixtures. The neutron scattering amplitude of a subunit for an arbitrary volume fraction $0 \leq x \leq 1$ of $\mathrm{D}_{2} \mathrm{O}$ in solution and subunit deuteration $0 \leq \mathrm{y} \leq 1$ can be expressed as $^{25}$ :

$$
A(x, y)=(1-x-y) A^{(00)}+x A^{(01)}+y A^{(10)},
$$

where $A^{(00)}, A^{(01)}$ and $A^{(10)}$ are the scattering amplitudes of protonated subunit in pure water, protonated subunit in $100 \% \mathrm{D}_{2} \mathrm{O}$ and of perdeuterated subunit in $\mathrm{H}_{2} \mathrm{O}$, respectively. Given this relation, scattering patterns from any combination of protonated or deuterated subunits with known structure can be readily computed and compared with the experimental data sets ( $\mathrm{see}^{25}$ for more details). 
Met/InlB complex in solution

Apart from fitting multiple X-ray and neutron experimental data SASREF allows one to also account for the known interfaces (e.g. binding sites) between subunits by restraining the corresponding inter-residue distances. The target function minimized in SASREF has the form:

$$
F=\frac{1}{N_{c}} \sum_{i=1}^{N_{c}} \chi_{i}^{2}+\text { penalties }
$$

The first term in (4) ensures minimization of the overall discrepancy in multiple data sets $\left(N_{c}\right.$ is the total number of scattering curves) from contrast variation series and/or from partial constructs, the second penalty term formulates various requirements and restrictions to the model. For neutron scattering, the computed theoretical curves from the model entering Eq. 2 are appropriately smeared by the instrument resolution function introduced by. ${ }^{48}$

The results of multiple SASREF runs were analyzed to determine common structural features using the programs DAMAVER ${ }^{29}$ and SUPCOMB. ${ }^{49}$ The latter program aligns two arbitrary low or high resolution models represented by ensembles of points by minimizing a dissimilarity measure called normalized spatial discrepancy (NSD). For every point (bead or atom) in the first model, the minimum value among the distances between this point and all points in the second model is found, and the same is done for the points in the second model. These distances are added and normalized against the average distances between the neighboring points for the two models. Generally, NSD values close to unity indicate that the two models are similar. The program DAMAVER generates the average model of the set of superimposed structures 
Met/InlB complex in solution

and also specifies the most typical model (i.e. that having the lowest average NSD with all the other models of the set).

The program OLIGOMER ${ }^{28}$ was employed to analyze the 1:1 mixture of Met $_{567}$ and $\mathrm{InlB}_{321}$. This program finds volume fractions of components to minimize the discrepancy (2) between the linear superposition of the weighted intensities of the components and the experimental data from the mixture. 
Met/InlB complex in solution

\section{Acknowledgments}

Sample deuteration was carried out in the joint ILL/EMBL Deuteration laboratory, a platform of the Partnership for Structural Biology (PSB; http://psb.esrf.fr/). We thank Sabine Schmidt for expert technical assistance and Dr. Roland Wagner, Nadine Konisch and Anja Kobold for fermentation of $\mathrm{CHO}$ cells. M.V.P. and D.I.S. acknowledge support from the EU Design Study "SAXIER”, contract number RIDS011934. This work was supported in part by the DFG priority program 1150 (to H.H.N). D.W.H. acknowledges support from the Fonds der Chemischen Industrie. 
Met/InlB complex in solution

\section{References}

1. Swaminathan, B. \& Gerner-Smidt, P. (2007). The epidemiology of human listeriosis. Microbes Infect. 10, 1236-1243.

2. Hamon, M., Bierne, H. \& Cossart, P. (2006). Listeria monocytogenes: a multifaceted model. Nat. Rev. Microbiol. 4, 423-434.

3. Gaillard, J. L., Berche, P., Frehel, C., Gouin, E. \& Cossart, P. (1991). Entry of $L$. monocytogenes into cells is mediated by internalin, a repeat protein reminiscent of surface antigens from gram-positive cocci. Cell $\mathbf{6 5}, 1127-1141$.

4. Mengaud, J., Ohayon, H., Gounon, P., Mege, R. M. \& Cossart, P. (1996). Ecadherin is the receptor for internalin, a surface protein required for entry of $L$. monocytogenes into epithelial cells. Cell 84, 923-932.

5. Dramsi, S., Biswas, I., Maguin, E., Braun, L., Mastroeni, P. \& Cossart, P. (1995). Entry of Listeria monocytogenes into hepatocytes requires expression of InIB, a surface protein of the internalin multigene family. Mol. Microbiol. 16, 251-261.

6. Shen, Y., Naujokas, M., Park, M. \& Ireton, K. (2000). InIB-dependent internalization of Listeria is mediated by the Met receptor tyrosine kinase. Cell $103,501-510$.

7. Birchmeier, C., Birchmeier, W., Gherardi, E. \& Vande Woude, G. F. (2003). Met, metastasis, motility and more. Nat. Rev. Mol. Cell Biol. 4, 915-925.

8. Maulik, G., Shrikhande, A., Kijima, T., Ma, P. C., Morrison, P. T. \& Salgia, R. (2002). Role of the hepatocyte growth factor receptor, c-Met, in oncogenesis and potential for therapeutic inhibition. Cytokine Growth Factor Rev. 13, 41-59. 
Met/InlB complex in solution

9. Schubert, W. D. \& Heinz, D. W. (2003). Structural aspects of adhesion to and invasion of host cells by the human pathogen Listeria monocytogenes. Chembiochem. 4, 1285-1291.

10. Banerjee, M., Copp, J., Vuga, D., Marino, M., Chapman, T., van der Geer, P. \& Ghosh, P. (2004). GW domains of the Listeria monocytogenes invasion protein InlB are required for potentiation of Met activation. Mol. Microbiol. 52, 257-271.

11. Braun, L., Nato, F., Payrastre, B., Mazie, J. C. \& Cossart, P. (1999). The 213amino-acid leucine-rich repeat region of the Listeria monocytogenes InlB protein is sufficient for entry into mammalian cells, stimulation of PI 3-kinase and membrane ruffling. Mol. Microbiol. 34, 10-23.

12. Jonquieres, R., Pizarro-Cerda, J. \& Cossart, P. (2001). Synergy between the Nand C-terminal domains of InlB for efficient invasion of non-phagocytic cells by Listeria monocytogenes. Mol. Microbiol. 42, 955-965.

13. Niemann, H. H., Jager, V., Butler, P. J., van den Heuvel, J., Schmidt, S., Ferraris, D., Gherardi, E. \& Heinz, D. W. (2007). Structure of the human receptor tyrosine kinase met in complex with the Listeria invasion protein InlB. Cell 130, 235-246.

14. Marino, M., Braun, L., Cossart, P. \& Ghosh, P. (1999). Structure of the lnlB leucine-rich repeats, a domain that triggers host cell invasion by the bacterial pathogen L. monocytogenes. Mol. Cell 4, 1063-1072.

15. Schubert, W. D., Gobel, G., Diepholz, M., Darji, A., Kloer, D., Hain, T., Chakraborty, T., Wehland, J., Domann, E. \& Heinz, D. W. (2001). Internalins from the human pathogen Listeria monocytogenes combine three distinct folds into a contiguous internalin domain. J. Mol. Biol. 312, 783-794. 
Met/InlB complex in solution

16. Marino, M., Banerjee, M., Jonquieres, R., Cossart, P. \& Ghosh, P. (2002). GW domains of the Listeria monocytogenes invasion protein InlB are SH3-like and mediate binding to host ligands. EMBO J. 21, 5623-5634.

17. Machner, M. P., Frese, S., Schubert, W. D., Orian-Rousseau, V., Gherardi, E., Wehland, J., Niemann, H. H. \& Heinz, D. W. (2003). Aromatic amino acids at the surface of InlB are essential for host cell invasion by Listeria monocytogenes. Mol. Microbiol. 48, 1525-1536.

18. Gherardi, E., Youles, M. E., Miguel, R. N., Blundell, T. L., Iamele, L., Gough, J., Bandyopadhyay, A., Hartmann, G. \& Butler, P. J. (2003). Functional map and domain structure of MET, the product of the c-met protooncogene and receptor for hepatocyte growth factor/scatter factor. Proc. Natl. Acad. Sci. U S A 100, $12039-12044$.

19. Stamos, J., Lazarus, R. A., Yao, X., Kirchhofer, D. \& Wiesmann, C. (2004). Crystal structure of the HGF beta-chain in complex with the Sema domain of the Met receptor. $E M B O J . \mathbf{2 3}, 2325-2335$.

20. Feigin, L. A. \& Svergun, D. I. (1987). Structure analysis by small-angle $x$-ray and neutron scattering, Plenum Press, New York.

21. Svergun, D. I. \& Koch, M. H. J. (2003). Small angle scattering studies of biological macromolecules in solution. Rep. Progr. Phys. 66, 1735-1782.

22. King, W. A., Stone, D. B., Timmins, P. A., Narayanan, T., von Brasch, A. A., Mendelson, R. A. \& Curmi, P. M. (2005). Solution structure of the chicken skeletal muscle troponin complex via small-angle neutron and X-ray scattering. $J$. Mol. Biol. 345, 797-815. 
Met/InlB complex in solution

23. Svergun, D. I. \& Nierhaus, K. H. (2000). A map of protein-rRNA distribution in the 70 S Escherichia coli ribosome. J. Biol. Chem. 275, 14432-14439.

24. Petoukhov, M. V. \& Svergun, D. I. (2005). Global rigid body modelling of macromolecular complexes against small-angle scattering data. Biophys. J. 89, 1237-1250.

25. Petoukhov, M. V. \& Svergun, D. I. (2006). Joint use of small-angle X-ray and neutron scattering to study biological macromolecules in solution. Eur. Biophys. J. 35, 567-576.

26. Gherardi, E., Sandin, S., Petoukhov, M. V., Finch, J., Youles, M. E., Ofverstedt, L. G., Miguel, R. N., Blundell, T. L., Vande Woude, G. F., Skoglund, U. \& Svergun, D. I. (2006). Structural basis of hepatocyte growth factor/scatter factor and MET signalling. Proc. Natl. Acad. Sci. U S A 103, 4046-4051.

27. Svergun, D. I., Barberato, C. \& Koch, M. H. J. (1995). CRYSOL - a program to evaluate X-ray solution scattering of biological macromolecules from atomic coordinates. J. Appl. Crystallogr. 28, 768-773.

28. Konarev, P. V., Volkov, V. V., Sokolova, A. V., Koch, M. H. J. \& Svergun, D. I. (2003). PRIMUS - a Windows-PC based system for small-angle scattering data analysis. J. Appl. Crystallogr. 36, 1277-1282.

29. Volkov, V. V. \& Svergun, D. I. (2003). Uniqueness of ab initio shape determination in small angle scattering. J. Appl. Crystallogr. 36, 860-864.

30. Schlessinger, J. (2000). Cell signaling by receptor tyrosine kinases. Cell 103, 211225. 
Met/InlB complex in solution

31. Michieli, P., Mazzone, M., Basilico, C., Cavassa, S., Sottile, A., Naldini, L. \& Comoglio, P. M. (2004). Targeting the tumor and its microenvironment by a dualfunction decoy Met receptor. Cancer Cell 6, 61-73.

32. Mattinen, M. L., Paakkonen, K., Ikonen, T., Craven, J., Drakenberg, T., Serimaa, R., Waltho, J. \& Annila, A. (2002). Quaternary structure built from subunits combining NMR and small-angle x-ray scattering data. Biophys. J. 83, 11771183.

33. Grishaev, A., Wu, J., Trewhella, J. \& Bax, A. (2005). Refinement of multidomain protein structures by combination of solution small-angle X-ray scattering and NMR data. J. Am. Chem. Soc. 127, 16621-16628.

34. Konarev, P. V., Petoukhov, M. V., Volkov, V. V. \& Svergun, D. I. (2006). ATSAS 2.1, a program package for small-angle scattering data analysis. J. Appl. Crystallogr. 39, 277-286.

35. Meilleur, F., Contzen, J., Myles, D. A. \& Jung, C. (2004). Structural stability and dynamics of hydrogenated and perdeuterated cytochrome P450cam (CYP101). Biochemistry 43, 8744-8753.

36. Artero, J.-B., Hartlein, M., McSweeney, S. \& Timmins, P. (2005). A comparison of refined X-ray structures of hydrogenated and perdeuterated rat y-crystallin in $\mathrm{H}_{2} \mathrm{O}$ and $\mathrm{D}_{2} \mathrm{O}$. Acta Crystallogr. D Biol. Crystallogr. 61, 1541-1549.

37. Boulin, C. J., Kempf, R., Gabriel, A. \& Koch, M. H. J. (1988). Data acquisition systems for linear and area X-ray detectors using delay line readout. Nucl. Instrum. Meth. A 269, 312-320. 
Met/InlB complex in solution

38. Koch, M. H. J. \& Bordas, J. (1983). X-ray diffraction and scattering on disordered systems using synchrotron radiation. Nucl. Instrum. Meth. 208, 461-469.

39. Gabriel, A. \& Dauvergne, F. (1982). The localization method used at EMBL. Nucl. Instrum. Meth. 201, 223-224.

40. Svergun, D. I. (1993). A direct indirect method of small-angle scattering data treatment. J. Appl. Crystallogr. 26, 258-267.

41. Guinier, A. (1939). La diffraction des rayons X aux tres petits angles; application a l'etude de phenomenes ultramicroscopiques. Ann. Phys. (Paris) 12, 161-237.

42. Svergun, D. I. (1992). Determination of the regularization parameter in indirect transform methods using perceptual criteria. J. Appl. Crystallogr. 25, 495 - 503.

43. Porod, G. (1982). General theory. In Small-angle X-ray scattering (Kratky, O., ed.), pp. 17-51. Academic Press, London.

44. Ghosh, R. E., Egelhaaf, S. \& Rennie, A. R. R. (2006). A computing Guide for Small Angle Scattering. In Internal ILL Report No. ILL06GH05T.

45. Jakob, C. G., Lewinski, K., Kuciel, R., Ostrowski, W. \& Lebioda, L. (2000). Crystal structure of human prostatic acid phosphatase. Prostate 42, 211-218.

46. Svergun, D. I., Barberato, C. \& Koch, M. H. J. (1995). CRYSOL - a program to evaluate X-ray solution scattering of biological macromolecules from atomic coordinates. J. Appl. Crystallogr. 28, 768-773.

47. Svergun, D. I., Richard, S., Koch, M. H., Sayers, Z., Kuprin, S. \& Zaccai, G. (1998). Protein hydration in solution: experimental observation by x-ray and neutron scattering. Proc. Natl. Acad. Sci. U S A 95, 2267-2272. 
Met/InlB complex in solution

48. Pedersen, J. S., Posselt, D. \& Mortensen, K. (1990). Analytical treatment of the resolution function for small-angle scattering. J. Appl. Crystallogr. 23, 321-333.

49. Kozin, M. B. \& Svergun, D. I. (2001). Automated matching of high- and lowresolution structural models. J. Appl. Crystallogr. 34, 33-41. 
Met/InlB complex in solution

\section{Figure Captions}

Fig. 1. Elution profile from a Superdex200 column of equimolar amounts of Met $_{928}$ or $\mathrm{InlB}_{321}$ alone (top left and right), of an equimolar mixture of the two proteins (bottom left) and of a mixture containing a 1:2 molar ratio of Met $_{928}$ and $\operatorname{InlB}_{321}$ (bottom right).

Fig. 2 Elution profile from a Superdex200 column of equimolar amounts of Met $_{567}$ or $\mathrm{InlB}_{321}$ alone (top left and right), of an equimolar mixture of the two proteins (bottom left) and of a mixture containing a 1:80 molar ratio of $\mathrm{Met}_{567}$ and $\mathrm{InlB}_{321}$ (bottom right).

Fig. 3. Solution scattering patterns of Met, its constructs and their mixtures with $\operatorname{InlB}_{321}$. (a) SAXS profiles of InlB 321, Met $_{567}$, Met $_{567}+\operatorname{InlB}_{321}$, Met $_{838}$, Met $_{928}$, Met $_{838}+\operatorname{InlB}_{321}$ and Met $_{928}+$ InlB $_{321}$ (see Table 3 for curve references). Experimental data are denoted by dots, the fits by CRYSOL for (1) and (2), OLIGOMER for (3) and SASREF for (4)-(7) are shown as solid lines. (b) SANS contrast variation of Met $_{838}$, Met $_{928}$ and their complexes with InlB $_{321}$. Experimental data are denoted by dots, the fits by SASREF are presented as solid lines.

Fig. 4. The best superposition of the Met-InlB rigid body model reconstructed from SAS data and the crystal structure of Met Sema, PSI and Ig1 in complex with InlB ${ }_{321}$. Sema, PSI, Ig1, Ig2, Ig3 and Ig4 domains are shown in gray, orange, red, purple, cyan and green respectively. $\mathrm{InlB}_{321}$ is presented in blue. The SAS model is shown as transparent surface 
Met/InlB complex in solution

representation in light colors, the crystal structure as ribbon representation in dark colors. Carbohydrates are not shown.

Fig. 5. Comparison of (a) the rigid body model of the Met-InlB complex reconstructed from SAS data and (b) the crystal structure of the Met $_{741}-\operatorname{InlB}_{321}$ complex with the refined position of $\operatorname{Ig} 2$ and added $\operatorname{Ig} 3$ and $\operatorname{Ig} 4$ domains to yield the best agreement with SAXS and SANS data. The color scheme is as in Fig. 4. Both structures are shown in surface representation including carbohydrates. 
Met/InlB complex in solution

Table 1. Parameters derived from size exclusion chromatography (SEC) and dynamic light scattering (DLS)

\begin{tabular}{|c|c|c|c|c|c|}
\hline & Calculated & & & & LS \\
\hline & $M M, \mathrm{kDa}$ & $V e, \mathrm{ml}$ & $M M, \mathrm{kDa}$ & $R h, \mathrm{~nm}$ & $M M, \mathrm{kDa}$ \\
\hline Met $_{567}$ & 62 & 12.82 & 80 & 3.5 & 60 \\
\hline Met $_{567}+\operatorname{InlB}_{321}$ & 94 & $12.86 / 14.44$ & $80 / 37$ & 3.3 & 52 \\
\hline $\operatorname{Met}_{741}$ & 82 & 11.85 & 129 & 4.5 & 111 \\
\hline $\operatorname{Met}_{741}+\operatorname{InlB}_{321}$ & 114 & 11.45 & 156 & 4.8 & 130 \\
\hline $\operatorname{Met}_{838}$ & 92 & 11.4 & 159 & 4.6 & 117 \\
\hline $\operatorname{Met}_{838}+\operatorname{InlB}_{321}$ & 124 & 11.09 & 186 & 5.0 & 143 \\
\hline Met $_{928}$ & 102 & 10.87 & 207 & 5.3 & 165 \\
\hline Met $_{928}+\operatorname{InlB}_{321}$ & 135 & 10.62 & 232 & 5.5 & 181 \\
\hline
\end{tabular}

$V e$ and $R h$ are, respectively, the elution volume from a Superdex200 HR10/30 column and the hydrodynamic radius. 
Met/InlB complex in solution

Table 2. Overall parameters derived from SAXS data

\begin{tabular}{|c|c|c|c|c|}
\hline Construct/parameter & $R_{g}, \mathrm{~nm}$ & $M M, \mathrm{kDa}$ & $V_{p}, \mathrm{~nm}^{3}$ & $D_{\max }, \mathrm{nm}$ \\
\hline $\mathrm{InlB}_{321}$ & $3.1 \pm 0.1$ & $30 \pm 5$ & $62 \pm 10$ & $10.0 \pm 0.5$ \\
\hline Met $_{567}$ & $3.2 \pm 0.1$ & $61 \pm 10$ & $120 \pm 10$ & $10.0 \pm 0.5$ \\
\hline Met $_{838}$ & $4.8 \pm 0.1$ & $102 \pm 10$ & $180 \pm 20$ & $15.5 \pm 0.5$ \\
\hline Met $_{928}$ & $4.9 \pm 0.1$ & $107 \pm 10$ & $210 \pm 20$ & $17.0 \pm 0.5$ \\
\hline $\operatorname{Met}_{567}+\operatorname{InlB} 321$ & $2.9 \pm 0.1$ & $43 \pm 5$ & $85 \pm 10$ & $10 \pm 0.5$ \\
\hline $\operatorname{Met}_{838}+\operatorname{InlB}_{321}$ & $4.6 \pm 0.1$ & $126 \pm 10$ & $240 \pm 20$ & $15.5 \pm 0.5$ \\
\hline Met $_{928}+\operatorname{InlB}{ }_{321}$ & $4.7 \pm 0.1$ & $131 \pm 10$ & $270 \pm 20$ & $17.0 \pm 0.5$ \\
\hline
\end{tabular}

$R_{g}, D_{\max }, V_{p}$ and $M M$ are, respectively, the radius of gyration, maximum size, excluded volume and molecular mass, calculated from the SAXS experimental data. 
Met/InlB complex in solution

Table 3. Summary of SAS measurements.

\begin{tabular}{|c|c|c|c|c|c|c|c|}
\hline Constructs / Experiments & X-rays & $\begin{array}{l}\text { Neutrons, } \\
0 \% \mathrm{D}_{2} \mathrm{O}\end{array}$ & $\begin{array}{l}\text { Neutrons, } \\
35 \% \mathrm{D}_{2} \mathrm{O}\end{array}$ & $\begin{array}{l}\text { Neutrons, } \\
50 \% \mathrm{D}_{2} \mathrm{O}\end{array}$ & $\begin{array}{l}\text { Neutrons, } \\
60 \% \mathrm{D}_{2} \mathrm{O}\end{array}$ & $\begin{array}{l}\text { Neutrons, } \\
81 \% \mathrm{D}_{2} \mathrm{O}\end{array}$ & $\begin{array}{l}\text { Neutrons, } \\
100 \% \mathrm{D}_{2} \mathrm{O}\end{array}$ \\
\hline & \multicolumn{7}{|c|}{ Contrast $\Delta \rho, 10^{10} \mathrm{~cm}^{-2}$} \\
\hline Protonated protein & 2.82 & 2.285 & 0.275 & -0.586 & -1.160 & -2.309 & -3.457 \\
\hline $50 \%$ deuterated protein & 2.82 & 4.652 & 2.643 & 1.781 & 1.207 & 0.059 & -1.090 \\
\hline \multirow[t]{2}{*}{ Perdeuterated protein } & 2.82 & 7.020 & 5.010 & 4.149 & 3.575 & 2.426 & 1.278 \\
\hline & \multicolumn{7}{|c|}{ List of measurements $\left.{ }^{*}\right)$} \\
\hline Protonated $\mathrm{InlB}_{321}$ & $(1), 1.6$ & - & - & - & - & - & - \\
\hline $\mathrm{Met}_{567}$ & $(2), 1.4$ & - & - & - & - & - & - \\
\hline Met $_{567}+$ protonated InlB 321 & $(3), 1.1$ & - & - & - & - & - & - \\
\hline Met $_{838}$ & $(4), 1.9$ & $(33), 0.9$ & - & $(34), 0.9$ & - & - & $(32), 5.0$ \\
\hline Met $_{928}$ & $(5), 2.0$ & $(31), 0.8$ & - & $(30), 1.8$ & - & $(29), 1.1$ & $(28), 2.0$ \\
\hline Met $_{838}+$ protonated InlB $_{321}$ & $(6), 2.8$ & - & - & - & - & - & - \\
\hline Met $_{928}+$ protonated InlB $_{321}$ & (7), 2.1 & $(27), 1.0$ & - & - & - & - & $(26), 3.3$ \\
\hline $\begin{array}{l}\text { Met }_{838}+50 \% \text { deuterated } \\
\text { InlB }_{321}\end{array}$ & - & $(25), 1.1$ & $(24), 0.5$ & - & (9), 1.7 & - & $(23), 2.6$ \\
\hline $\begin{array}{l}\text { Met }_{928}+50 \% \text { deuterated } \\
\text { InlB }_{321}\end{array}$ & - & $(22), 1.5$ & $(21), 0.7$ & $(20), 0.6$ & - & $(19), 1.9$ & $(18), 3.1$ \\
\hline Met $_{838}+$ perdeuterated InlB $_{321}$ & - & $(17), 1.7$ & $(16), 1.1$ & - & $(8), 1.0$ & - & $(15), 2.5$ \\
\hline Met $_{928}+$ perdeuterated InlB $_{321}$ & - & $(14), 1.6$ & $(13), 1.4$ & $(12), 1.4$ & $(35), 1.3$ & $(11), 4.1$ & $(10), 4.8$ \\
\hline
\end{tabular}


Met/InlB complex in solution

$\left.{ }^{*}\right)$ The numbers in brackets corresponding to the numbering of curves in Fig. 3 are followed by the $\chi$ value. Symbol "-" means that the given construct was not measured at this condition. 

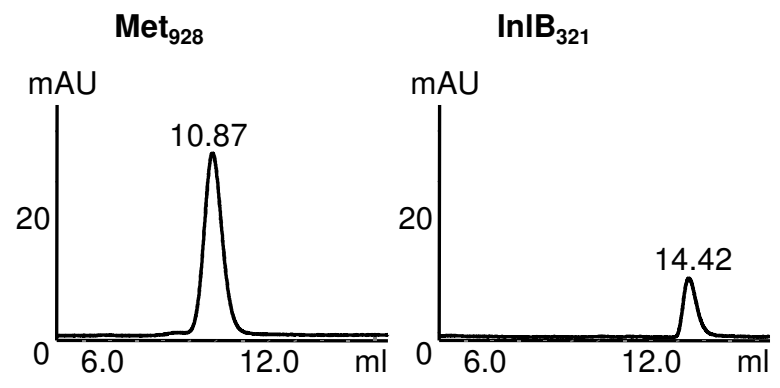

Met $_{928}: \operatorname{InIB}{ }_{321}$

Met $_{928}: \ln \mid B_{321}$

1:1

$1: 2$
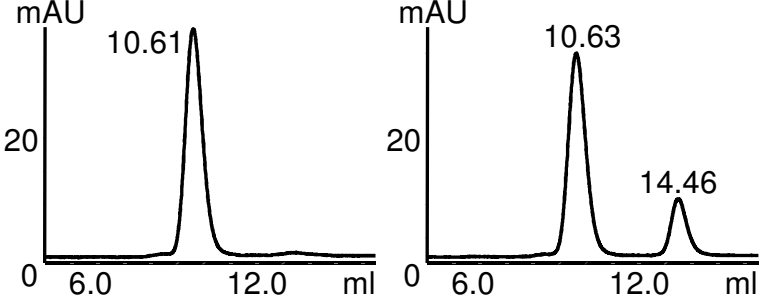


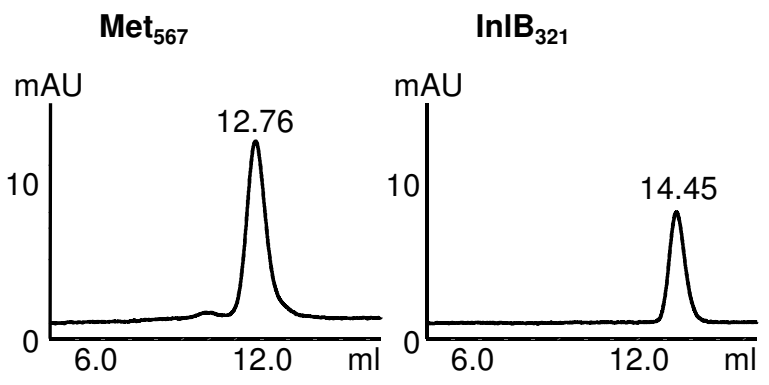

Met $_{567}:$ InIB 321

Met $_{567}: \operatorname{InI} \mathrm{B}_{321}$

1:1

$1: 80$

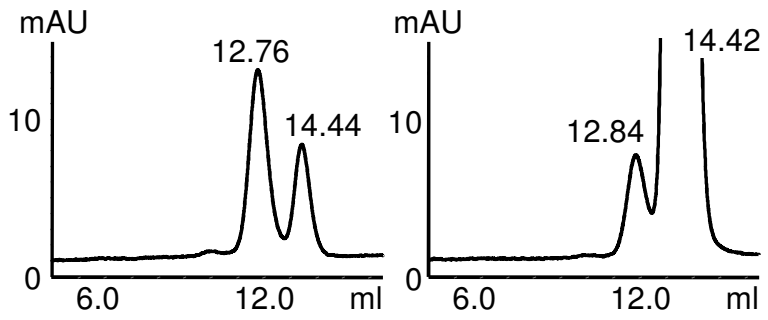


Ig I, relative

(a)

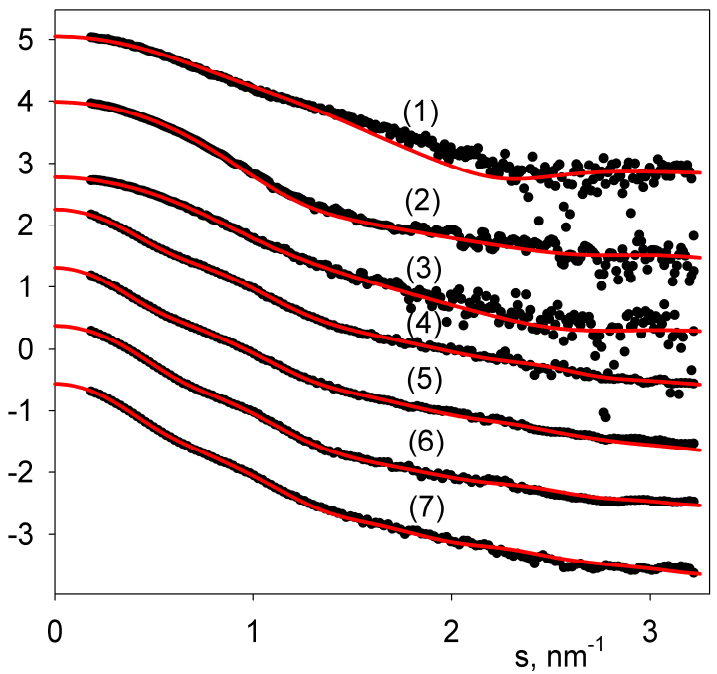

Ig I, relative

(b)

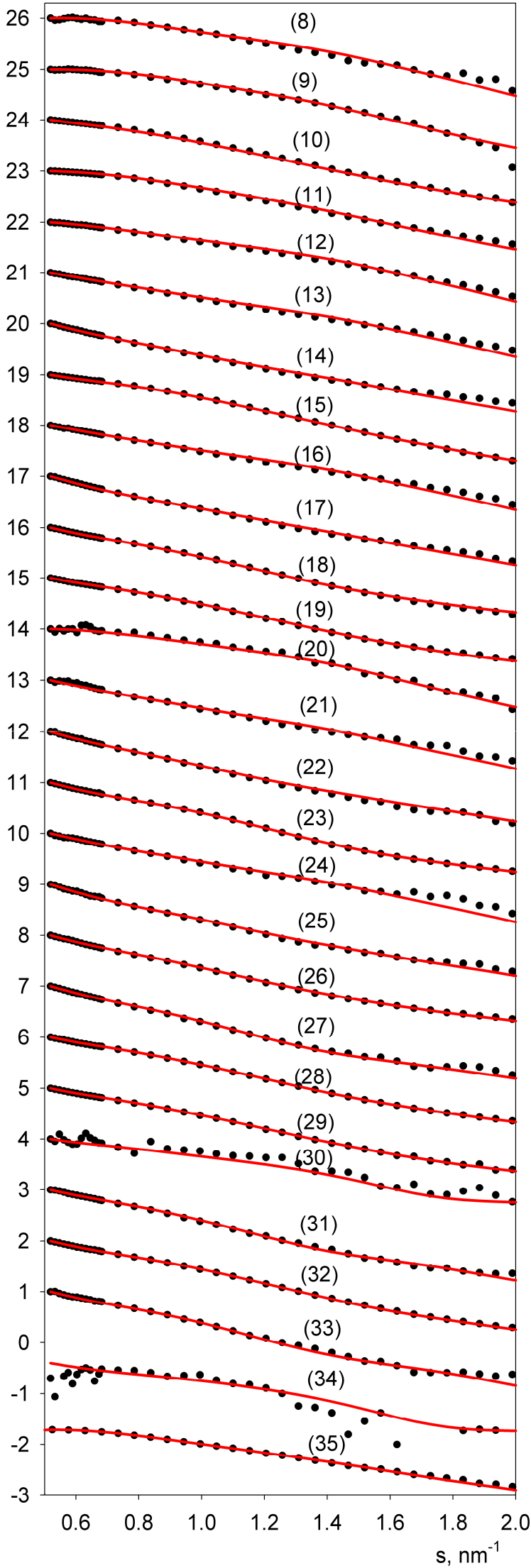




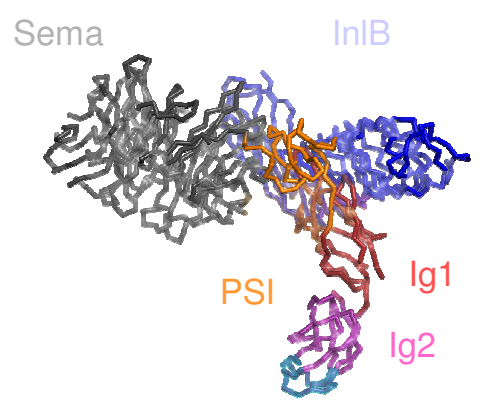

$\lg 3$

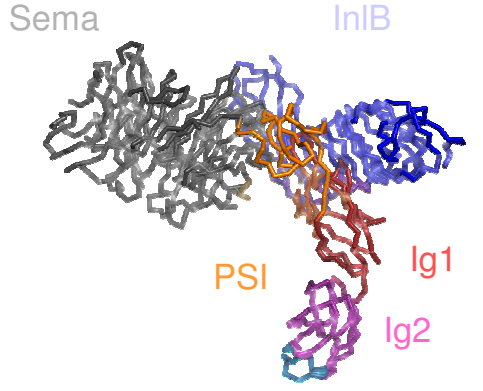

$\lg 3$

$\lg 4$ 
(a)
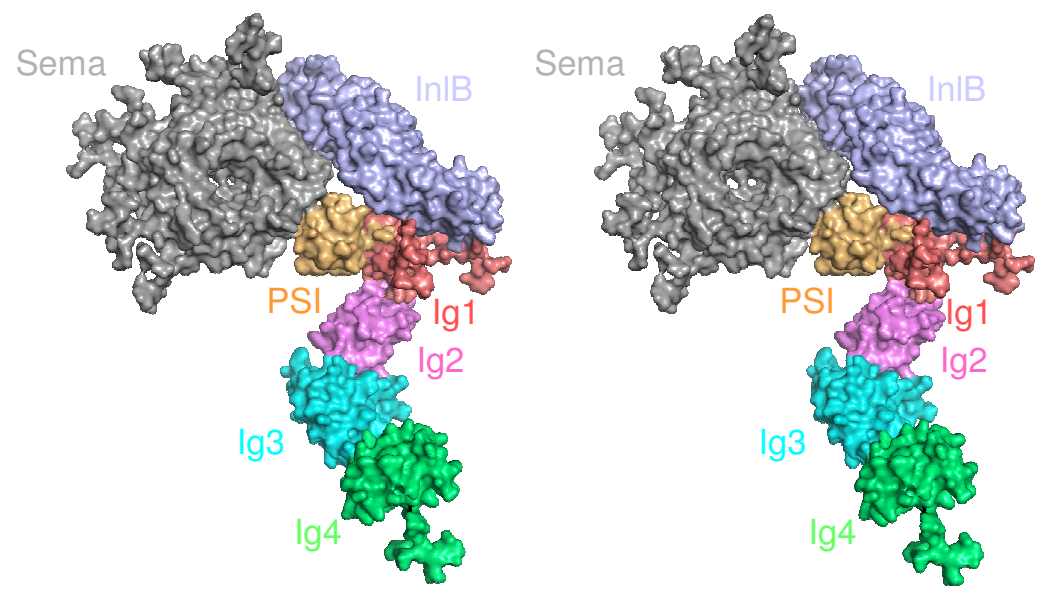

(b)

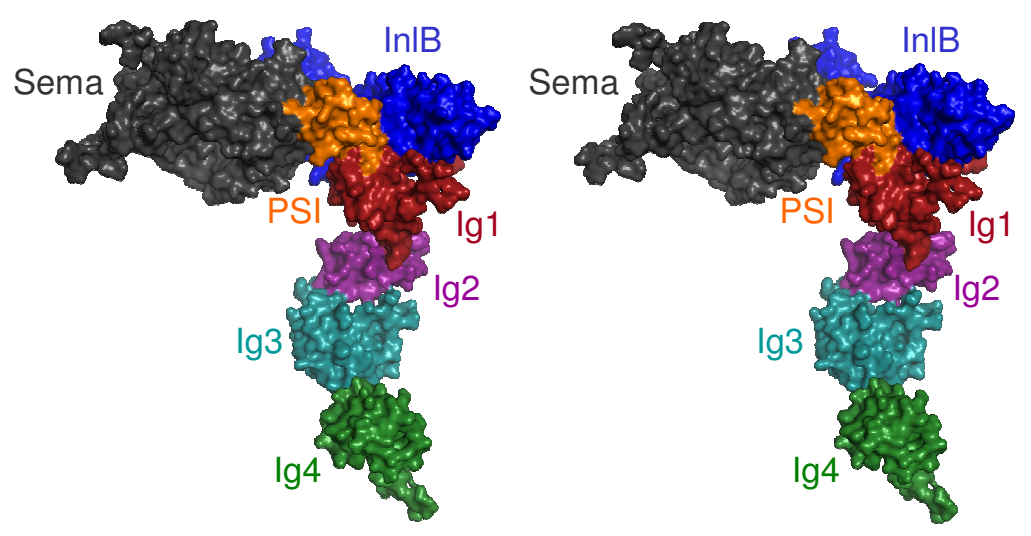

